\section{ORIGINAL RESEARCH}

F.M. Kovacs

E. Arana

A. Royuela

A. Estremera

G. Amengual

B. Asenjo

H. Sarasíbar

I. Galarraga

A. Alonso

C. Casillas

A. Muriel

C. Martínez

V. Abraira,

for the Spanish Back

Pain Research Network

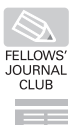

\title{
Vertebral Endplate Changes Are Not Associated with Chronic Low Back Pain among Southern European Subjects: A Case Control Study
}

\author{
BACKGROUND AND PURPOSE: Data on the association between vertebral endplate changes and low \\ back pain are contradictory. This study was designed to assess whether this association exists among \\ Southern European subjects.
}

MATERIALS AND METHODS: Patients in this study serving as cases were 35-50 years of age with low back pain lasting $>90$ days, for whom a lumbar MR imaging had been prescribed. Controls were subjects 35-50 years of age, having a cranial MR imaging for headache with normal findings, and no history of clinically relevant LBP. Two hundred forty cases and 64 controls were recruited consecutively in the radiology services across 6 cities in Spain. Imaging findings and subject characteristics were gathered through previously validated instruments. Radiologists who interpreted MRI were blinded to the subject characteristics. A multivariate logistic regression model was developed to assess the association of vertebral endplate changes with LBP, adjusting for sex, age, body mass index, lifetime exposure to smoking, physical activity, disk degeneration, and the interaction between disk degeneration and vertebral endplate changes.

RESULTS: Vertebral endplate changes were found in $80.4 \%$ of the cases and in $87.5 \%$ of the controls. In the regression model, disk degeneration was the only variable showing a confounding effect. Results showed that after adjusting for disk degeneration, the presence of vertebral endplate changes is associated with the absence of chronic LBP (OR for LBP: 0.31; 95\% Cl, 0.10-0.95).

CONCLUSIONS: In Southern European subjects, vertebral endplate changes are not associated with chronic LBP.

ABBREVIATIONS: $\mathrm{BMI}=$ body mass index; $\mathrm{Cl}=$ confidence interval; $\mathrm{LBP}=$ low back pain; $\mathrm{OR}=$ odds ratio; $\mathrm{RMQ}=$ Roland-Morris Questionnaire; $\mathrm{VAS}=$ visual analog scale

$\mathbf{L}^{\mathrm{B}}$ $\mathrm{BP}$ is defined as pain between the costal margins and the inferior gluteal folds, which may be associated with pain referred down to the leg (leg pain) and is usually accompanied by painful limitation of movement. Diagnosing common LBP implies that the pain is not related to conditions such as frac-

\section{Received October 12, 2011; accepted after revision November 25}

From the Departamento Científico (F.M.K.) and Spanish Back Pain Research Network (F.M.K., E.A., A.R., A.E., G.A., B.A., H.S., I.G., A.A., C.C., A.M., C.M., V.A.), Fundación Kovacs, Palma de Mallorca, Spain; Servicio de Radiología (E.A.), Fundación Instituto Valenciano de Oncología, Valencia, Spain; Fundación Instituto de Investigación en Servicios de Salud (E.A.), Valencia, Spain; Consorcio de Investigación Biomédica en Red especializado en Epidemiología y Salud Pública (A.R., V.A.), Instituto de Salud Carlos III, Madrid, Spain; Unidad de Bioestadística Clínica (A.R., H.S., A.M., V.A.), Hospital Ramón y Cajal, Madrid, Spain; Hospital Son Llàtzer, (A.E., G.A., C.M.), Palma de Mallorca, Spain; Hospital Carlos Haya (B.A.), Málaga, Spain; Hospital de Manacor (I.G.), Mallorca, Spain; Fundación Jiménez Díaz Avda (A.A.), Madrid, Spain; and Instituto de Traumatología Unión de Mutuas (C.C.), Castellón, Spain.

Francisco M. Kovacs reports that he had full access to all of the data in the study and takes responsibility for the integrity of the data and the accuracy of the data analysis.

This work was funded by the Kovacs Foundation, a not-for-profit Spanish institution specializing in neck and back pain research and with no links to the health industry.

The funding institution was not involved in the design and conduction of the study; data collection; management, analysis, and interpretation of the data; preparation, review, and approval of the manuscript; or in the decision to submit the article for publication.

No benefits in any form have been or will be received from a commercial party related directly or indirectly to the subject of this article. The authors do not have any financial or personal relationships with third parties that could influence this work inappropriately. The authors have no conflicts of interest to report.

Please address correspondence to Estanislao Arana, MD, Fundación Instituto Valenciano de Oncología, C/Beltrán Báguena, 19, 46009 Valencia, Spain; e-mail: aranae@uv

Indicates article with supplemental on-line table.

http://dx.doi.org/10.3174/ajnr.A3087 tures, direct trauma, or systemic diseases, such as spondylodiskitis or neoplastic, infectious, vascular, metabolic, or endocrine-related processes. ${ }^{1}$

In certain cases of LBP in which pain radiates down the leg and in which signs and symptoms correlate with radiologic findings, disk protrusion, disk herniation, and spinal stenosis are considered to be plausible causes of pain. ${ }^{1}$ However, these cases represent $<5 \%$, and the rest are considered to be "nonspecific," implying that no structural cause is triggering the pain. In "nonspecific" cases, pain is thought to originate in soft tissues. ${ }^{1}$

Vertebral endplate changes are defined as changes in the signal intensity of vertebral endplates and subchondral bone. ${ }^{2}$ Three different types have been described. Type I (low T1 and high T2 signals) is thought to reflect an ongoing active degenerative process, type II (high T1 and T2 signals) is believed to correspond to a more stable chronic degeneration, and type III (low T1 and T2 signals) is considered to be a sign of subchondral bone sclerosis. ${ }^{2}$

Vertebral endplate changes, also called "Modic" changes, ${ }^{3,4}$ have been observed in MR images of patients with $\mathrm{LBP}^{3,4}$ and have therefore been suspected to be a potential cause of "nonspecific" LBP, especially in chronic cases. "Chronic" cases are defined as those in which LBP lasts for $\geq 12$ weeks. ${ }^{5}$

However, vertebral endplate changes have also been observed in MR imaging performed in the general population ${ }^{6,7}$ and asymptomatic subjects. ${ }^{8-10}$ These are more likely to be found among men, especially as the subject gets older and signs of disk degeneration appear. ${ }^{11,12}$

It is currently debatable whether vertebral endplate changes 
are a cause of pain or just a clinically irrelevant radiologic finding. ${ }^{13-15}$ In fact, some studies have found an association between these changes and LBP, whereas others have not. Case control studies would be appropriate to study such an association, ${ }^{7}$ but are rarely reported in the spine literature. ${ }^{15}$ In fact, no case control study has assessed the association between vertebral endplate changes and low back pain, to our knowledge.

If vertebral endplate changes were actually associated with chronic LBP or could be seen as a cause of it, it would make sense for future studies to delve further into their potential prognostic value, to explore whether patients with such changes require specific treatment, or to establish whether their presence should imply any other variations to clinical management. However, if vertebral endplate changes were to be casual clinically irrelevant findings, common among asymptomatic subjects such as patients with LBP, the need to report these or to modify a patient's clinical management, depending on their presence, would be questionable. Therefore, the purpose of the present study was to assess whether vertebral endplate changes are associated with chronic LBP among Southern European subjects.

\section{Materials and Methods}

This was a case control study conducted in 6 hospitals across 6 cities in Spain. This study protocol was approved by the institutional review boards of the participating hospitals and was registered at the ClinicalTrials.gov data base (identifier NCT00479063).

\section{Subjects}

All subjects screened for this study were 35-50 years of age and had been prescribed an MR imaging. Patients had been referred for a lumbar MR imaging for LBP lasting $\geq 90$ days. Controls were patients with headache who had been referred for a cranial MR imaging, which turned out to have normal findings, and who either had no history of LBP or had only experienced 1 episode in their life, which had lasted for $<7$ days.

Exclusion criteria were ethnicity not Spanish, history of spine surgery, currently pregnant, scoliosis with $>15^{\circ}$ curvature, vertebral fractures, "red flags" for fractures or potential underlying systemic diseases, ${ }^{1}$ and signs suggesting cauda equina syndrome. ${ }^{1}$

When this study was designed, no data on the prevalence of vertebral endplate changes among Spanish asymptomatic subjects and patients with chronic LBP were available. Therefore, available data from studies conducted in the Northern European and Anglo-Saxon environments were used to calculate sample size. ${ }^{13}$

Sample size was established at 236 cases and 59 controls (total, 295 subjects) on the basis of the following assumptions: prevalence of vertebral endplate changes at $40 \%$ among patients with $\mathrm{LBP}^{13}$ and $19 \%$ among controls, $\alpha$ error of .05, statistical power of $90 \%$, and a ratio of cases per controls of 4:1.

\section{Procedure}

All the subjects who complied with inclusion criteria and signed the informed consent for participating in the study were included. Neither subjects nor physicians received compensation for participating in this study.

Controls were invited to participate once the recruiting radiologist had established that the cranial MR imaging had normal findings. For controls, participating in the study implied undergoing a lumbar MR imaging. This study did not require any further modifications to patient clinical management.
All the subjects underwent a lumbar MR imaging. Once the images had been obtained, auxiliary personnel provided the subjects with a self-administered questionnaire to gather demographic and clinical data and assigned a code number to each one. At the analysis phase, this code made it possible to match each subject's clinical data and MR imaging.

Each subject completed a self-administered questionnaire. Once completed, the auxiliary personnel added the code assigned to the subject and sent the questionnaire to a central coordination office. At the coordination office, data were introduced into a data base by 2 administrative assistants separately, who double-checked that the data introduced coincided with the questionnaires.

The auxiliary personnel sent the research coordinator a list indicating the code number assigned to each subject. For ethical reasons and following Spanish law on patient privacy and management of clinical data for research purposes, the research coordinator (F.M.K.) substituted another name on each image by the code assigned to the subject. Once subject identities had been masked on the images, he distributed these among participating radiologists, who read them at their own institutions.

At the beginning of the study, the research coordinator (F.M.K.) had established with the auxiliary personnel a key, within the patient's code, making it possible to distinguish cases from controls. The key was communicated to the statisticians when they started data analysis. The key remained unknown for the rest of the research staff, including radiologists. The research coordinator did not interpret any images.

This procedure ensured the following: 1) Radiologists were unaware of whether the images they were reading corresponded to a "case" or a "control," 2) they were blinded to subject demographic and clinical characteristics, 3 ) the only researchers who were aware of the code identifying each as a "case" or a "control" (the research coordinator and the statisticians) did not read the images, and 4) only the auxiliary personnel and the statisticians who conducted the analysis had access to subject demographic and clinical data.

\section{Clinical Assessment}

The self-administered questionnaire gathering demographic and clinical data included age (date of birth), sex, height (centimeters), weight (kilograms), lifetime smoking exposure (pack-years smoked), duration of pain (asking separately whether pain had lasted for $\geq 90$ days and the date of appearance), degree of physical activity, current pain severity, and current degree of disability. Physical activity was assessed through a previously validated questionnaire. ${ }^{16}$ Pain intensity was measured with a 10-cm VAS and LBP-related disability, with the validated version of the RMQ. ${ }^{17,18}$ Values, ranging from better to worse, are $0-10$ for VAS and 0-24 for RMQ. ${ }^{17,18}$

Subjects completed all the self-administered questionnaires by themselves, in private. The only instructions they received were those included in the validated instruments measuring physical activity, pain intensity, and disability. ${ }^{16-18}$ They received no help or additional instructions from health care personnel, research staff, or other third parties.

\section{MR Imaging Evaluation}

Eleven practicing general radiologists, working in 6 general hospitals located in 5 different geographic regions in Spain, participated in this study. Their postresidency experience as radiologists ranged from 12 to 18 years, and their experience interpreting spine imaging ranged between 7 and 12 years. They were trained in different institutions without fellowships. Their interobserver agreement when reporting 


\section{Protocol}

All examinations were performed on six 1.5T MR imaging systems with a 6-channel phased array spine coil. The following sequences were used without fat suppression:

- A localizer sequence of 5 images, $30 \mathrm{~ms} / 10 \mathrm{~ms} / 50^{\circ}$ (TR/TE/flip angle) consisting of 2 coronal and 3 sagittal images in orthogonal planes

- Sagittal T1-weighted spin-echo, 440-550 ms/14-20 ms (TR/TE), 156-307 × 192-512 matrix, 270-mm FOV, 11 sections of 4-mm thickness, 2 acquisitions

- Sagittal T2-weighted turbo spin-echo 2896-3300 ms/102-120 ms (TR/TE eff $_{1}$, 156-307 × 192-512 matrix, 270-mm FOV, 11 sections of 4-mm thickness, 2 acquisitions, echo-train length of 12

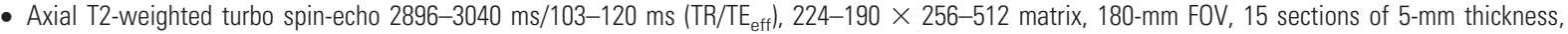
3 acquisitions, echo-train length of 5 . Sections were placed in the plane of the 5 lower disks

Note:- $\mathrm{TE}_{\text {eff }}$ indicates effective echo-time.

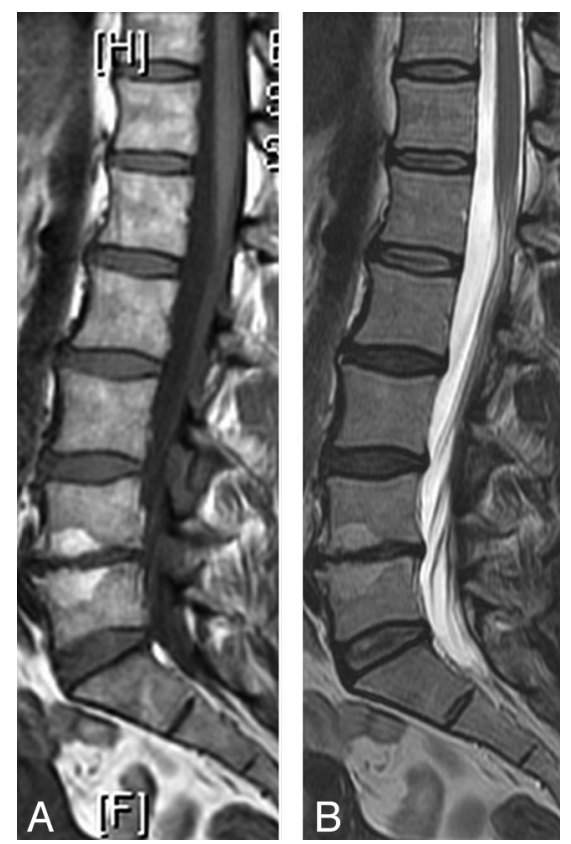

Fig 1. Modic II from a 50-year-old asymptomatic subject who was included in the control group. Sagittal T1- $(A)$ and T2-weighted $(B)$ images show hyperintensity in both endplates of the L4-L5 space, associated with signs of disk degeneration.

vertebral endplate changes had previously been shown to be better than that for any other radiologic finding. ${ }^{19-21}$

The methods for MR imaging evaluation used in this study were consistent with those used in studies focusing on radiologist agreement when interpreting lumbar MR imaging and specifically vertebral endplate changes. ${ }^{19-22}$ All examinations were performed on six 1.5T MR imaging systems with a 6-channel phased array spine coil and with patients in the supine position, following an imaging protocol (Table 1 and Fig 1). Findings on MR imaging were reported by using the previously validated Spanish version of the "Nordic Modic Consensus Group Classification," ${ }^{19-22}$ which includes grading of disk degeneration according to the classification of Pfirrmann et al, ${ }^{23}$ from grade I (normal) to grade $\mathrm{V}$ (most advanced degree of disk degeneration), and data on the type and location of vertebral endplate changes.

\section{Data Analysis}

At the analysis phase, the degree of physical activity was classified as "high intensity" versus "non-high intensity" "; lifetime exposure to smoking was defined as the number of packs smoked during the patient's lifetime. ${ }^{14}$ Patients with any vertebral endplate change at any vertebral level were classified as "showing vertebral endplate changes," irrespective of the type or location; disk degeneration was dichotomized into grades I + II + III ("no/mild disk degeneration") versus IV $+\mathrm{V}$ (severe disk degeneration).
Absolute and relative frequencies were used to describe categoric variables. For continuous ones, medians and interquartile range or mean and SD were used, depending on whether data were normally distributed.

The $\chi^{2}$ test was used to compare the proportion of subjects showing vertebral endplate changes between cases and controls. To control for potential confounders, we performed a multivariate logistic regression to estimate the association between vertebral endplate changes and chronic LBP. Based on previous studies, the variables, which were included in the maximal model, were age (years), sex, BMI (kilogram per square meter), lifetime exposure to smoking (pack-years smoked), exposure to high-intensity physical activity, severe disk degeneration, and the interaction between disk degeneration and vertebral endplate changes. ${ }^{6,12,15,24}$

We used a backward strategy; those variables that changed $\geq 10 \%$ of the effect size when they were eliminated from the model were considered as confounders. The collinearity of the maximal models was evaluated by using the criteria proposed by Belsley, ${ }^{25}$ and the significance level was set at $5 \%$.

The STATA (Version 11.0; StataCorp, College Station, Texas) program was used for analysis.

\section{Results}

Ten radiologists from the 6 participating hospitals screened 64 subjects without LBP and 287 patients with LBP with a lumbar MR imaging. Among the latter, 47 were excluded for pain lasting $<90$ days. Therefore, 304 subjects were included, 240 cases and 64 controls.

Among the 240 patients with LBP who were included, the median (P25, P75) age was $43(38-47)$ years, and $132(55.0 \%)$ were women. Among the 64 subjects without LBP, the median age was 45 (41-47) years, and 29 (45.3\%) were women. Table 2 details sample characteristics. There were some missing values, due to questions patients left unanswered in the questionnaires.

The question left unanswered by more subjects was the one requesting the date of appearance of pain; among the 240 patients with chronic LBP, 55 left it unanswered. Because it was hypothesized that differences between patients who did and did not answer this question might exist, their characteristics were compared (On-line Table 1 ) and a sensitivity analysis was performed, in which these 55 cases were excluded. Characteristics of patients who answered and did not answer this question were very similar (On-line Table 1). Therefore, according to the parsimony principle, ${ }^{26}$ only results from the analysis including all the recruited subjects are shown (Table 3 ).

At least 1 vertebral endplate change (of any type and loca- 


\begin{tabular}{|c|c|c|c|c|}
\hline Variable & No. $^{a}$ & $\begin{array}{l}\text { Patients with LBP } \\
\text { (240 Subjects) }\end{array}$ & No. $^{a}$ & $\begin{array}{l}\text { Subjects without LBP } \\
\text { (64 Subjects) }\end{array}$ \\
\hline Age (range) $(y r)^{b}$ & 240 & $43(38-47)$ & 64 & $45(41-47)$ \\
\hline $\operatorname{Sex}^{\mathrm{C}}$ & 240 & & 64 & \\
\hline Male (median) & & $108(45)$ & & $35(54.7)$ \\
\hline Female (median) & & $132(55)$ & & $29(45.3)$ \\
\hline BMI (range) $\left(\mathrm{Kg} / \mathrm{m}^{2}\right)^{\mathrm{b}}$ & 228 & $24.6(22.4-27.3)$ & 60 & $25.2(21.9-27.4)$ \\
\hline Lifetime smoking (pack-years) (range) ${ }^{b}$ & 224 & $2.3(0.0-11.2)$ & 60 & $3(0-11)$ \\
\hline Highly intense physical activity (median) ${ }^{c}$ & 199 & $51(25.6)$ & 48 & $9(18.8)$ \\
\hline Low back pain severity (VAS) (range) ${ }^{\mathrm{b}}$ & 229 & $4(2-7)$ & 52 & $0(0-0)$ \\
\hline Duration of pain (range) (mo) & 185 & $46(11-112)$ & 64 & \\
\hline Disability (range) (RMO) ${ }^{\mathrm{b}}$ & 213 & $6(3-12)$ & 6 & $1(1-1)$ \\
\hline Vertebral endplate changes (any type $)^{\mathrm{c}, \mathrm{d}}$ & 240 & $193(80.4)$ & 64 & 56 (87.5) \\
\hline Types of vertebral endplate changes ${ }^{\mathrm{C}}$ & 193 & & 56 & \\
\hline Only type I & & $10(4.2)$ & & $1(1.6)$ \\
\hline Types I and || & & $38(15.8)$ & & $14(21.9)$ \\
\hline Types I and III & & $2(0.8)$ & & $0(0.0)$ \\
\hline Type I, II, and III & & $11(4.6)$ & & $0(0.0)$ \\
\hline Only type II & & $120(50.0)$ & & $40(62.5)$ \\
\hline Types II and III & & $11(4.6)$ & & $0(0.0)$ \\
\hline Only type III & & $1(0.4)$ & & $1(1.6)$ \\
\hline No. of vertebral endplate changes per patient ${ }^{\mathrm{b}}$ & 240 & $2(1 ; 3)$ & 64 & $3(2 ; 4)$ \\
\hline Severe disk degeneration ${ }^{\mathrm{c}}$ & 240 & $158(65.8)$ & 64 & $30(46.9)$ \\
\hline
\end{tabular}

${ }^{a}$ No. of subjects in each group for whom data on the corresponding variable were available (subjects who answered the corresponding question or in whom the variable was applicable - eg, age, sex, weight, height, patients who showed any type of vertebral endplate change or severe disk degeneration, and so forth).

b Median (P25, P75).

${ }^{\mathrm{c}}$ No. $(\%)$.

${ }^{d} P$ value for the comparison of the proportion of vertebral endplate changes among subjects without LBP, compared with those with LBP: .191.

Table 3: Results from the regression model on the association between vertebral endplate changes and chronic LBP, in which all patients with LBP (240) were included ${ }^{\mathrm{a}}$

\begin{tabular}{|c|c|c|c|c|}
\hline & \multicolumn{2}{|c|}{ Crude Analysis } & \multicolumn{2}{|c|}{ Adjusted Analysis } \\
\hline & OR $(95 \% \mathrm{CI})$ & $P$ & OR $(95 \% \mathrm{CI})^{\mathrm{b}}$ & $P$ \\
\hline Vertebral endplate changes & $0.43(0.14-1.29)$ & .132 & $0.31(0.10-0.95)$ & .041 \\
\hline
\end{tabular}

tion) was present in $87.5 \%$ of the 64 subjects without LBP and in $80.4 \%$ of the 240 patients with LBP $(P=.191)$ (Table 2$)$.

In the regression model, crude analyses showed no association between vertebral endplate changes and LBP, and only disk degeneration was found to have a confounding effect. After adjusting for this variable, results showed that the odds ratio $(95 \% \mathrm{CI})$ for those with chronic low back pain when having any type of vertebral endplate change was $0.30(0.10-$ $0.95)$. The association between vertebral endplate changes and the absence of chronic low back pain reached marginal statistical significance $(P=.041)$ (Table 3$)$.

The results from sensitivity analysis, in which only the 185 patients who provided data on the exact duration of pain were included, were very similar, though the association between vertebral endplate changes and the absence of chronic low back pain did not reach statistical significance $(P=.120)$. In this analysis, in addition to disk degeneration, age and highly intense physical activity showed a confounding effect. After adjusting for these variables, the OR (95\% CI) was 0.39 (0.12-1.28).

\section{Discussion}

Results from this study suggest that, among Spanish subjects, vertebral endplate changes are not associated with chronic
LBP. In fact, vertebral endplate changes were more common among controls (Table 2) and were associated with absence of chronic LBP (Table 3).

Several factors may explain the inconsistencies between results from previous studies assessing the association between the existence of vertebral endplate changes and LBP. First, given that genetic differences influence disk degeneration, ${ }^{27,28}$ which is the factor most strongly associated with vertebral endplate changes, ${ }^{12}$ genetic differences may also influence the prevalence and clinical relevance of such vertebral changes and might explain variations across studies conducted with different populations. In fact, the only previous study reporting the prevalence of vertebral endplate changes among Southern European (Spanish) patients with LBP found it to be $81 \%$, with a 95\% CI, 77\%-85\%. ${ }^{12}$ This prevalence is higher than those reported in other geographic settings, ${ }^{4,6-10,13}$ but virtually identical to the one found in the current study (Table 2).

Second, if vertebral endplate changes are actually not associated with LBP, as this study suggests, inconsistencies could also be explained by fortuitous differences between the characteristics of each study sample, such as age, disk degeneration, genetic profile, or other unknown confounders. For instance, in this study, the exclusion of 55 cases for the sensitivity analysis sufficed for the association of vertebral endplate changes and absence of LBP to cease to be significant, despite the fact that the characteristics of the subjects included were very similar to those excluded (On-line Table 1). Third, differences in results across studies may also derive from differences in sample characteristics, study design, and methods. ${ }^{3,7,13,29-31}$ For instance, results may be influenced by whether there were the following conditions: 1) Asymptomatic subjects were included, 2 ) imaging reading was blinded to patient clinical characteristics, 3) reporting followed a previously validated nomenclature and protocol, 4) images were 
interpreted by several radiologists, 5) radiologists were blinded to other radiologists' interpretations, 6) the interobserver agreement of participating radiologists had been previously assessed and shown to be acceptable, and 7) potential confounders were accounted for at the analysis phase.

Type II is the most prevalent vertebral endplate change $e^{2-4,6-12}$ and the one that most subjects in this study had, either isolated or in combination with type I. This study was designed to assess the association between LBP and any type of vertebral endplate change. Assessing its association with any particular characteristic (eg, type or affected vertebral volume) would have required a different design, with different inclusion criteria, comparison groups, and sample size. Probably because of the 4:1 ratio between cases and controls, some uncommon combinations were more prevalent among the former (eg, isolated type I or combinations of types I/III, II/III, and I/II/III). However, this possibility is not likely to alter the conclusion of this study because a recent systematic review found no differences in the association between LBP and any particular type of vertebral endplate change. ${ }^{13}$

It is unlikely that a "causal," "predisposing," or "triggering" factor will not show an "association" with its corresponding condition. However, because the current study was crosssectional and had a case control design, it might be argued that it does not rule out the possibility of vertebral endplate changes influencing the prognosis of LBP. ${ }^{7,32}$ This is not likely because among asymptomatic subjects, presenting with a type I vertebral endplate change does not increase the risk of developing LBP, ${ }^{32}$ vertebral endplate changes do not influence the clinical course of back pain, and they are not a prognostic factor for recovery. ${ }^{33}$ Nevertheless, further prospective studies might assess whether subjects with vertebral endplate changes show different responsiveness to certain specific forms of treatment, such as exercise or some types of surgery.

The characteristics and representativeness of cases and controls are essential in any case control study. ${ }^{15}$ Age influences the prevalence of vertebral endplate changes, ${ }^{6,9,11,12,14}$ as well as LBP. ${ }^{2}$ Therefore, subjects had to be between 35 and 50 years of age to ensure the comparability of age across controls and cases. In this study, controls were subjects without LBP and without a meaningful history of LBP. For ethical and operational reasons, they were recruited among subjects with normal findings on cranial MR imaging, which had been requested for an unrelated condition (headache). Because the lifetime prevalence of LBP is $>70 \%,{ }^{1}$ at the design phase, it was considered unrealistic to exclude all the subjects reporting having had a single short ( $<7$ days) LBP episode and they were accepted as controls. This does not contradict the conclusion of this study; if vertebral endplate changes were to be associated with a single short episode of LBP in patients' lifetimes, they could be considered as clinically irrelevant.

"Cases" were not defined as "patients with LBP " but specifically as "chronic patients in whom LBP was serious enough to prescribe a lumbar MR imaging." These are likely to be patients the clinicians perceive as more serious or those in whom LBP does not improve despite treatment. ${ }^{34}$ Chronic patients cause the major clinical, social, and economic burden deriving from $\mathrm{LBP}^{1,35,36}$ and represent the population in which vertebral endplate changes were more likely to play a causal role. Subjects were recruited consecutively, and all those who complied with inclusion criteria were included. Moreover, the prevalence found among Spanish patients with chronic LBP in this study is very similar to the one from the only previous study conducted in this geographic setting. ${ }^{12}$ All these suggest that the generalizability of the results from the current study to middle-aged Spanish patients with chronic LBP is not a major concern.

Results from the current study further support the poor correlation between clinical symptoms and radiologic findings in patients with LBP and the notion that imaging is only appropriate in a specific subset of patients with LBP in whom results may actually change clinical management. ${ }^{37,38}$

A growing body of evidence questions the effectiveness of spinal fusion ${ }^{39,40}$ and suggests that complex instrumented fusion may cause unnecessary harm to patients. ${ }^{41,42}$ However, some surgical societies continue to recommend such a surgery on the basis of the existence of vertebral endplate changes and other radiologic findings. ${ }^{43}$ Therefore, unless future highquality studies show that vertebral endplate changes are indeed clinically relevant, it may be sound to consider changing the way radiologists report findings on lumbar MR imaging, as was the case for plain radiography of the spine, ${ }^{44}$ moving away from describing anatomic features to focusing on findings that are more likely to be clinically relevant, such as nerve compression. ${ }^{45,46}$ In the case of vertebral endplate changes, refraining from including this finding in radiologic reports or mentioning it as a finding that is associated with disk degeneration but is likely to be clinically irrelevant may be a way of protecting patients from unnecessarily aggressive forms of treatment or overtreatment. $^{43}$

This study has some weaknesses. Fifty-five $(22.9 \%)$ of the 240 LBP patients recruited as "cases" did not answer the question on the date when pain appeared. Therefore, although they were chronic (ie, their pain had lasted for $>90$ days), the exact duration of their pain could not be calculated. It might be argued that these 55 patients are likely to correspond to those who had LBP longer so that it was more difficult for them to recall exactly when the pain appeared. It might also be hypothesized that the 185 patients who provided the date of appearance of pain had an incident to which they attributed pain, though there is no evidence to support this because according to the definition of "LBP" that was used for this study, patients with any direct trauma or "red flags" for fracture were excluded. ${ }^{1}$ However, patients who did and did not provide these data were very similar (On-line Table 1) and results remained consistent in the sensitivity analysis, which only included patients for whom the exact duration of pain was known. Therefore, any hypothetic differences between these subsets of patients do not question the conclusions from this study. In fact, the main difference between results from the 2 analyses is that in the sensitivity one, the association between vertebral endplate changes and the absence of pain did not reach statistical significance, probably due to the loss of statistical power caused by the reduction in sample size.

The reliability of the assessment of lumbar MR imaging is poor for spinal stenosis; fair for osteophytes; and moderate for vertebral endplate changes, Schmorl nodes, disk degeneration, annular tears, and disk contour. ${ }^{19-21,47}$ However, this limitation is inherent to the use of lumbar MR imaging ${ }^{21,23,48}$ and does not challenge the clinical applicability of these results. 
This study also has some strengths: The definition of "case" corresponded to the type of patients with LBP in whom vertebral endplate changes were more prevalent and more likely to be clinically relevant ${ }^{3,4,26}$; cases and controls were recruited in the same setting ${ }^{15}$; the agreement in the reporting of vertebral endplate changes among radiologists interpreting MRI had been shown to be satisfactory by using the methods and imaging protocol implemented in this study ${ }^{19-21}$; radiologists were blinded to subject characteristics (including whether they were "cases" or "controls"); imaging findings were reported through a previously validated classification ${ }^{49}$; and results were adjusted for potential confounders. ${ }^{12-15}$

\section{Conclusions}

These results suggest that in Spanish subjects, the existence of vertebral endplate changes in the lumbar spine is not associated with chronic low back pain.

\section{References}

1. Waddell G. The Back Pain Revolution. London, UK: Churchill-Livingstone; 2004:1-61

2. Modic MT, Steinberg PM, Ross JS, et al. Degenerative disk disease: assessment of changes in vertebral body marrow with MR imaging. Radiology 1988;166:193-99

3. Rahme R, Moussa R. The Modic vertebral endplate and marrow changes: pathologic significance and relation to low back pain and segmental instability of the lumbar spine. AJNR Am J Neuroradiol. 2008;29:838-42

4. Kuisma M, Karppinen J, Niinimaki J, et al. Modic changes in endplates of lumbar vertebral bodies: prevalence and association with low back and sciatic pain among middle-aged male workers. Spine (Phila Pa 1976) 2007;32: $1116-22$

5. Merskey HB, Bogduk N. Detailed descriptions of pain syndromes. In: Merskey HB, Bogduk N, eds. Classification of Chronic Pain. Seattle: IASP Press; 1994:39-58

6. Jensen TS, Bendix T, Sorensen JS, et al. Characteristics and natural course of vertebral endplate signal (Modic) changes in the Danish general population. BMC Musculoskelet Disord 2009;10:81

7. Kjaer P, Leboeuf-Yde C, Korsholm L, et al. Magnetic resonance imaging and low back pain in adults: a diagnostic imaging study of 40-year-old men and women. Spine (Phila Pa 1976) 2005;30:1173-80

8. Weishaupt D, Zanetti M, Hodler J, et al. Painful lumbar disk derangement: relevance of endplate abnormalities at MR imaging. Radiology 2001;218:420-27

9. Chung CB, Vande Berg BC, Tavernier T, et al. End plate marrow changes in the asymptomatic lumbosacral spine: frequency, distribution and correlation with age and degenerative changes. Skeletal Radiol 2004;33:399-404

10. Kanayama M, Togawa D, Takahashi C, et al. Cross-sectional magnetic resonance imaging study of lumbar disc degeneration in 200 healthy individuals. J Neurosurg Spine 2009;11:501-07

11. Albert HB, Briggs AM, Kent P, et al. The prevalence of MRI-defined spinal pathoanatomies and their association with Modic changes in individuals seeking care for low back pain. Eur Spine J 2011;20:1355-62

12. Arana E, Kovacs FM, Royuela A, et al. Modic changes and associated features in Southern European chronic low back pain patients. Spine J 2011;11:402-11

13. Jensen TS, Karppinen J, Sorensen JS, et al. Vertebral endplate signal changes (Modic change): a systematic literature review of prevalence and association with non-specific low back pain. Eur Spine J 2008;17:1407-22

14. Jensen TS, Kjaer P, Korsholm L, et al. Predictors of new vertebral endplate signal (Modic) changes in the general population. Eur Spine J 2010;19:129-35

15. Pearson AM, Tosteson TD, Weinstein JN. The importance of study design in the spine literature. Semin Spine Surg 2009;21:202-09

16. Kuorinka I, Jonsson B, Kilbom A, et al. Standardised Nordic questionnaires for the analysis of musculoskeletal symptoms. Appl Ergon 1987;18:233-37

17. Huskisson EC. Measurement of pain. Lancet 1974;2:1127-31

18. Kovacs FM, Llobera J, Gil Del Real MT, et al. Validation of the Spanish version of the Roland-Morris questionnaire. Spine (Phila Pa 1976) 2002;27:538-42

19. Kovacs FM, Royuela A, Jensen TS, et al. Agreement in the interpretation of magnetic resonance images of the lumbar spine. Acta Radiol 2009;50:497-506

20. Arana E, Royuela A, Kovacs FM, et al. Agreement in the interpretation of 1.5T magnetic resonance images of the lumbar spine using the Nordic Modic Consensus Group Classification form. Radiology 2010;254:809-17

21. Arana E, Kovacs FM, Royuela A, et al. The influence of nomenclature in the interpretation of lumbar disk contour on MRI: a comparison of the agreement using the Combined Task Force and the Nordic nomenclatures. AJNR Am J Neuroradiol 2011;32:1143-48
22. Jensen TS, Sorensen JS, Kjaer P. Intra- and interobserver reproducibility of vertebral endplate signal (Modic) changes in the lumbar spine: the Nordic Modic Consensus Group classification. Acta Radiol 2007;48:748-54

23. Pfirrmann CW, Metzdorf A, Zanetti M, et al. Magnetic resonance classification of lumbar intervertebral disc degeneration. Spine (Phila Pa 1976) 2001;26: 1873-78

24. Videman T, Battie MC, Parent E, et al. Progression and determinants of quantitative magnetic resonance imaging measures of lumbar disc degeneration: a five-year follow-up of adult male monozygotic twins. Spine (Phila Pa 1976) 2008;33:1484-90

25. Belsley DA. Conditioning Diagnostics: Collinearity and Weak Data in Regression. New York: John Wiley \& Sons; 1991 tern Med 2011;154:181-89

26. Harrell FE. Regression Modeling Strategies with Applications to Linear Models, Logistics Regression, and Survival Analysis. New York: Springer-Verlag; 2001

27. Jim JJ, Noponen-Hietala N, Cheung KM, et al. The TRP2 allele of COL9A2 is an age-dependent risk factor for the development and severity of intervertebral disc degeneration. Spine (Phila Pa 1976) 2005;30:2735-42

28. Battie MC, Videman T, Kaprio J, et al. The Twin Spine Study: contributions to a changing view of disc degeneration. Spine J 2009;9:47-59

29. Quattrocchi CC, Alexandre AM, Pepa GM, et al. Modic changes: anatomy, pathophysiology and clinical correlation. Acta Neurochir Suppl 2011;108: $49-53$

30. Lim CH, Jee WH, Son BC, et al. Discogenic lumbar pain: association with MR imaging and CT discography. Eur J Radiol 2005;54:431-37

31. Fayad F, Lefevre-Colau MM, Rannou F, et al. Relation of inflammatory Modic changes to intradiscal steroid injection outcome in chronic low back pain. Eur Spine J 2007;16:925-31

32. Jarvik JG, Hollingworth W, Heagerty PJ, et al. Three-year incidence of low back pain in an initially asymptomatic cohort: clinical and imaging risk factors. Spine (Phila Pa 1976) 2005;30:1541-49

33. Keller A, Boyle E, Skog TA, et al. Are Modic changes prognostic for recovery in a cohort of patients with non-specific low back pain? Eur Spine J 2012;21:418-24

34. Kovacs FM, Fernandez C, Cordero A, et al. Non-specific low back pain in primary care in the Spanish National Health Service: a prospective study on clinical outcomes and determinants of management. BMC Health Serv Res 2006;6:57

35. Andersson GB. Epidemiologic features of chronic low-back pain. Lancet 1999;354:581-85

36. van Tulder MW, Koes BW, Bouter LM. A cost-of-illness study of back pain in The Netherlands. Pain 1995;62:233-40

37. Endean A, Palmer KT, Coggon D. Potential of magnetic resonance imaging findings to refine case definition for mechanical low back pain in epidemiological studies: a systematic review. Spine (Phila Pa 1976) 2011;36:160-69

38. Chou R, Qaseem A, Owens DK, et al. Clinical Guidelines Committee of the American College of Physicians diagnostic imaging for low back pain: advice for high-value health care from the American College of Physicians. Ann Intern Med 2011;154:181-89

39. Chou R, Qaseem A, Snow V, et al. Diagnosis and treatment of low back pain: a joint clinical practice guideline from the American College of Physicians and the American Pain Society. Ann Intern Med 2007;147:478-91

40. Chou R, Loeser JD, Owens DK, et al. Interventional therapies, surgery, and interdisciplinary rehabilitation for low back pain: an evidence-based clinical practice guideline from the American Pain Society. Spine (Phila Pa 1976) 2009;34:1066-77

41. Martin BI, Mirza SK, Comstock BA, et al. Are lumbar spine reoperation rates falling with greater use of fusion surgery and new surgical technology? Spine (Phila Pa 1976) 2007;32:2119-26

42. Wilson-MacDonald H, Fairbank J, Frost H, et al. The MRC Spine Stabilization Trial: surgical methods, outcomes, costs, and complications of surgical stabilization. Spine (Phila Pa 1976) 2008;33:2334-40

43. American Association of Neurological Surgeons. Blue Cross Blue Shield of North Carolina Lumbar Spine Fusion Surgery "Notification": www.spine.org/ Documents/BCBSNC_Lumbar_Fusion_Response121510.pdf. Published December 15, 2010. Updated December 15, 2010. Accessed June 30, 2011

44. Roland M, van Tulder M. Should radiologists change the way they report plain radiography of the spine? Lancet 1998;352:229-30

45. Carragee EJ, Alamin TF, Miller JL, et al. Discographic, MRI and psychosocial determinants of low back pain disability and remission: a prospective study in subjects with benign persistent back pain. Spine J 2005;5:24-35

46. Weishaupt D, Zanetti M, Hodler J, et al. MR imaging of the lumbar spine: prevalence of intervertebral disk extrusion and sequestration, nerve roo compression, end plate abnormalities, and osteoarthritis of the facet joints in asymptomatic volunteers. Radiology 1998;209:661-66

47. Carrino JA, Lurie JD, Tosteson ANA, et al. Lumbar spine: reliability of MR imaging findings. Radiology 2009;250:161-70

48. Jarvik JG, Deyo RA. Moderate versus mediocre: the reliability of spine MR data interpretations. Radiology 2009;250:15-17

49. Benoist M. The Michel Benoist and Robert Mulholland yearly European Spine Journal Review: a survey of the "medical" articles in the European Spine Journal, 2009. Eur Spine J 2010;19:3-10 